Methods it applies a multivariable longitudinal categorical and continuous analysis using Cox proportional hazards models. This analysis used data from the Bambui Health and Ageing Study, conducted in Bambui city in southeastern Brazil. The study population comprised 1512 (86.8\%) of all eligible 1742 elderly residents. Results In multivariable analysis using sleep duration as categorical variable and controlling for multiple measures of sociodemographic and health status those who slept nine hours or more per night were found to be at higher risk of mortality than those who slept seven hours. Excluding those whose deaths occurred within two years after entry, this association remained significant. In analyses using sleep duration as a continuous variable a linear correlation was found between sleep duration and mortality in all adjusted models in the whole sample and following exclusion of those whose deaths occurred within 2 year after entry. Both linear and quadratic terms were significant reflecting a predicted relationship, with mortality predominantly increasing in association with long sleep duration but with the addition of a slight decrease in association with shorter sleep duration.

Conclusion Long sleep duration rather than short sleep duration was principally associated with all-cause mortality in this sample. It is therefore reasonable to suggest that clinicians should be aware of the potential adverse prognosis associated with prolonged sleep.

\section{P2-38 TOOTH LOSS AND ITS ASSOCIATION WITH OBESITY IN AN ADULT POPULATION OF BRAZIL}

doi:10.1136/jech.2011.142976h.74

L M Pilotto, R K Celeste, ${ }^{*}$ S M B de Slavutsky, E Faerstein. UFRGS, Porto Alegre, Rio Grande do Sul, Brazil

Obesity and dental caries are important public health problems worldwide and are both associated with many adverse health outcomes. Identify if any of these problems leads to another is important to prioritise prevention actions and direct public policy. The aim of this study was to evaluate the association between body mass index and tooth loss in an adult population in Rio de Janeiro (Pró-Saúde Study), Brazil. Cross-sectional data were collected through self-administered questionnaire and anthropometric measurements were taken from 3930 technical-administrative staff of the university. Self-reported tooth loss (four categories) was the outcome and obesity was the main exposure variable. Data on aspects of diet, access and utilisation of healthcare, socioeconomic factors, health behaviours, and demographics were used as covariates to control for potential confounding. Compared to those with $\mathrm{BMI}<25$, overweight people $(\mathrm{BMI}>25 \&<30)$ showed a higher chance of tooth loss ( $\mathrm{OR}=1.66,95 \% \mathrm{CI} 1.41$ to 1.95$)$ and obese people (BMI>30) showed a $\mathrm{OR}=2.04$ (95\% CI 1.67 to 2.48). Adjusting for diet, access and use of services, health behaviours and socio economic overweight and obese subjects continued with a statistically significant OR of tooth loss. However, this association lost statistical significance after adjusted for weight stability since individuals were 20 years old=overweight individuals showed an adjusted $\mathrm{OR}=1.10$ ( $95 \%$ CI 0.78 to 1.55 ) and obese individuals an $\mathrm{OR}=1.17$ (95\% CI 0.85 to 1.62 ). This was a surprise because we were exploring the possibility of decay lead to tooth loss and is to be inferred in obesity. More efforts are needed to elucidate this issue.

\section{P2-39 SECONDHAND SMOKE EXPOSURE IN CHILDREN IS ASSOCIATED WITH COMMON CAROTID ARTERY INTIMA- MEDIA THICKNESS}

doi:10.1136/jech.2011.142976h.75

${ }^{1,2} \mathrm{~J}$ O'Loughlin, ${ }^{1,2} \mathrm{~B}$ Lauzon, ${ }^{3} \mathrm{~J}$ Dubois, ${ }^{4} \mathrm{G}$ Paradis, ${ }^{5} \mathrm{~K}$ Maximova, ${ }^{1,6} \mathrm{~S}$ Cengelli, ${ }^{*}$ ${ }^{7} \mathrm{~A}$ Tremblay, ${ }^{2,3} \mathrm{M}$ Lambert. ${ }^{1}$ Centre de Recherche du CHUM, Montreal, Quebec,
Canada; ${ }^{2}$ Université de Montréal, Montreal, Quebec, Canada; ${ }^{3}$ Sainte-Justine Hospital, Montreal, Quebec, Canada; ${ }^{4}$ Mc Gill University, Montreal, Quebec, Canada; ${ }^{5}$ University of Alberta, Alberta, Canada; ' Université de Lausanne, Lausanne, Vaud, Switzerland; ${ }^{7}$ Université Laval, Quebec, Quebec, Canada

Objective To investigate the association between common carotid artery intima-media thickness (cIMT) and exposure to secondhand smoke (SHS) in children.

Methods Data were available at baseline in the Quebec Adiposity and Lifestyle investigation in Youth (QUALITY) study, an ongoing longitudinal investigation of Caucasian children aged 8-10 years at cohort inception, who had at least one obese parent. Data on exposure to parents, siblings and friends smoking were collected in interviewer-administered child, and self-report parent questionnaires. Blood cotinine was measured with a high sensitivity ELISA. cIMT was measured by ultrasound. The association between blood cotinine and cIMT was investigated in multivariable linear regression analyses controlling for age, body mass index, and child smoking status.

Results Mean (SD) cIMT (0.5803 (0.04602)) did not differ across age or sex. Overall $26 \%, 6 \%$ and $3 \%$ of children were exposed to parents, siblings and friends smoking, respectively. Cotinine ranged from $0.13 \mathrm{ng} / \mathrm{ml}$ to $7.38 \mathrm{ng} / \mathrm{ml}$ (median $(\mathrm{IOR})=0.18 \mathrm{ng} / \mathrm{ml}$ )). Multivariately, a $1 \mathrm{ng} / \mathrm{ml}$ increase in cotinine was associated with a $0.090 \mathrm{~mm}$ increase in cIMT $(\mathrm{p}=0.034)$.

Conclusion In children as young as age 8-10 years, exposure to SHS relates to cIMT, a marker of pre-clinical atherosclerosis. Given the wide range of health effects of SHS, increased public health efforts are needed to reduced exposure among children in homes an private vehicles.

\section{P2-40 PREDICTORS OF SMOKING CESSATION IN ADOLESCENT SMOKERS: A SYSTEMATIC REVIEW OF LONGITUDINAL STUDIES}

doi:10.1136/jech.2011.142976h.76

${ }^{1,2} \mathrm{~S}$ Cengelli, ${ }^{2}{ }^{2,3} \mathrm{~J}$ O'Loughlin, ${ }^{2,3} \mathrm{~B}$ Lauzon, 'J Cornuz. 'Université de Lausanne, Lausanne, Vaud, Switzerland; ${ }^{2}$ Centre de Recherche du CHUM (CRCHUM), Montreal, Quebec, Canada; ${ }^{3}$ Université de Montréal, Montreal, Quebec, Canada

Tobacco use causes more than 5 million deaths worldwide annually. In Canada (2009), prevalence of smoking was $13 \%$ among those $15-19$ years and $23 \%$ among those 20-24 years. Many young smokers desire to quit, but have difficulty doing so. Empirical reviews have concluded that smoking cessation programs in youth have limited efficacy. In order to provide a solid knowledge base for tobacco interventions, determinants of self-initiated cessation in youth need to be understood. We systematically searched PUBMED and EMBASE for longitudinal studies on determinants of self-initiated smoking cessation in youth. $\mathrm{N}=3807$ titles and $\mathrm{N}=787$ abstracts were reviewed independently by two and three reviewers, respectively. Inclusion criteria were: published between January 1984 and August 2010, youth 10-28 years, and smoking cessation of $\geq 6$ months. Seven articles were retained for in-depth analysis. 3 of 7 studies retained defined smoking cessation as abstinence of $\geq 6$ months and four studies as 12 months. Seven factors emerged related to quitting: few friends who smoke, no intention to smoke, higher parental education, intact nuclear family, parental disapproval of smoking, good grades, good health, high cigarette resistance selfefficacy, and older age at first use. Additional factors are significant only in some studies or only assessed once. The longitudinal literature on predictors of youth cessation is not well developed. The most consistent predictors of self-initiated cessation include few friends smoking and no intention to smoke in the future. Tobacco interventions should target youth as well their friends as soon as possible after smoking onset given the difficulty in quitting. 